Casals R. et al.. (0000) "h-Principle for 4-dimensional Contact Foliations,"

International Mathematics Research Notices, Vol. 0000, Article ID rnn000, 31 pages.

doi:10.1093/imrn/rnn000

\title{
h-Principle for 4-dimensional Contact Foliations
}

\section{Roger Casals ${ }^{1}$, Álvaro del Pino ${ }^{1}$, and Francisco Presas ${ }^{2}$}

${ }^{1}$ Universidad Autónoma de Madrid and Instituto de Ciencias Matemáticas - CSIC. C. Nicolás Cabrera, 13-15, 28049, Madrid, Spain. ${ }^{2}$ Instituto de Ciencias Matemáticas - CSIC. C. Nicolás Cabrera, 13-15, 28049, Madrid, Spain.

Correspondence to be sent to: fpresas@icmat.es

In this article we introduce the topological study of codimension-1 foliations which admit contact structures on the leaves. A parametric existence $\mathrm{h}$-principle for foliated contact structures is provided for any cooriented foliation in a closed oriented $4-$ fold.

\section{Introduction}

In the present article we study the contact topology of the leaves of a codimension- 1 foliation in a given manifold. We introduce the definitions and objects of interest, detail their basic properties and explain related constructions. This article establishes the foundations of foliated contact topology. In particular, we also discuss some notions regarding symplectic foliations, and we show that contact and symplectic foliations are closely related, just as their non-foliated counterparts. The main result is a parametric existence $\mathrm{h}$-principle for foliated contact 4-folds.

Let us discuss briefly the non-foliated case. Symplectic and contact structures give rise to geometries in which there is a balance between flexible and rigid phenomena. A first instance of flexibility is stability under compact deformations: given a path of contact structures - or symplectic structures in the same cohomology class they are all isotopic. This constrasts sharply with the case of Riemannian or Poisson structures, which are fragile under perturbations - although stability statements can be made under certain hypotheses, see [Crainic, Fernandes, 2010]. In the same spirit of flexibility, contact and symplectic structures are rich in automorphisms:

Received

Communicated by

(c) The Author 0000. Published by Oxford University Press. All rights reserved. For permissions, please e-mail: journals.permissions@oxfordjournals.org. 
the automorphism group is an infinite dimensional Lie group, whereas, most times, Riemannian and Poisson structures have discrete automorphism groups.

Existence h-principles for contact structures in the open case [Eliashberg, Mishachev, 2002][Gromov, 1986] and the existence of a flexible class in the closed case [Eliashberg, 1989][Borman, Eliashberg, Murphy, 2014] are deeper instances of flexibility. Their parametric versions essentially state that, in those cases, the contact structure is not only stable under deformations that stay within the contact class, but also stable - in a certain sense - in the bigger, purely algebraic topological, class of almost contact structures -hyperplane distributions with an almost complex structure.

On the other hand, there is an h-principle for symplectic structures in open manifolds [Gromov, 1986], but not in closed ones. For instance, it is known that the extension to the interior of a ball of a germ of a symplectic structure defined along its boundary can be obstructed [Gromov, 1985]. We would say this is a rigidity phenomenon, because even when the purely homotopical problem can be solved - extending the germ to the interior just as an almost complex structure - the geometrical one might not be - extending it as an actual symplectic form.

In the same spirit of rigidity, much like interesting statements can be made for geodesic flows in Riemannian geometry, rigid dynamical behaviours are known to occur in the contact and symplectic worlds. See, for instance, Arnold's Conjecture in the symplectic case [Fukaya, Ono, 1999][Liu et al., 1998] and Weinstein's Conjecture in the contact case [Taubes, 2007][Weinstein, 1978]. Further, relevant geometric structures have been found in the contact and symplectic automorphism groups, see [Eliashberg, Kim, Polterovich, 2006][Polterovich, 2001], that state that they are very far - in general - from the diffeomorphism group.

These features establish contact topology and symplectic topology as fields in their own right within the realm of differential topology. Similarly, one would expect contact and symplectic foliations to display a wealth of interesting phenomena making them worth studying as particular subclasses of foliations. Indeed, symplectic foliations have been previously studied in the context of Poisson geometry [Libermann, 1983][Xu, 1992][Mikami, 2000][Abouqateb, Boucetta, 2003][Guillemin, Miranda, Pires, 2011] - they correspond precisely to regular Poisson structures and there are flexibility results concerning their existence. For instance, the article [Bertelson, 2002] extends Gromov's symplectic h-principle to open foliations. The corresponding h-principles for regular Poisson structures and $b$-Poisson structures have also been studied in [Frejlich, Fernandes, 2012] and [Frejlich, Martinez Torres, Miranda, 2013], respectively. In this vein, the present article presents a flexibility theorem for foliated contact structures in 4 -folds.

Let us state the main result. For this, we set up the following notation. The pair $(V, \mathcal{F})$ denotes a smooth, 
oriented, closed manifold $V$ endowed with a regular, cooriented, codimension-1 foliation $\mathcal{F}$. (This pair is fixed along the article.) Note that a manifold $V$ admits such a foliation if and only if $\chi(V)=0$, confer [Thurston, 1976, Theorem 1.A]. Consider the space $\mathcal{P}(V, \mathcal{F})$ of codimension-2 distributions $\xi$ on $V$ such that $\xi \subset T \mathcal{F}$, endowed with the compact-open topology. Define the following two spaces:

$$
\begin{aligned}
\mathcal{C}(V, \mathcal{F}) & =\{\xi \in \mathcal{P}(V, \mathcal{F}): \xi \text { induces a contact structure on each leaf of } \mathcal{F}\}, \\
\mathcal{A}(V, \mathcal{F}) & =\left\{(\xi, J): \xi \in \mathcal{P}(V, \mathcal{F}), J \in \operatorname{End}(\xi), J^{2}=-i d \text { and } J \text { compatible with } \xi\right\} .
\end{aligned}
$$

The elements in $\mathcal{C}(V, \mathcal{F})$ are called contact foliations (or foliated contact structures), and those in $\mathcal{A}(V, \mathcal{F})$ almost contact foliations (or foliated almost contact structures).

The space of compatible almost complex structures for a fixed contact structure is contractible, hence there exist maps $\pi_{k} \iota: \pi_{k} \mathcal{C}(V, \mathcal{F}) \rightarrow \pi_{k} \mathcal{A}(V, \mathcal{F})$ between homotopy groups. The study of $\mathcal{A}(V, \mathcal{F})$ lies within algebraic topology, whereas the understanding of the space of contact structures $\mathcal{C}(V, \mathcal{F})$ requires geometry.

In 3-dimensional contact topology there are no (geometric) obstructions to the existence of a contact structure in a given 3-fold [Martinet, 1971][Lutz, 1970]. The main result of this article states that the obstructions for the existence of a codimension- 1 contact foliation are also strictly topological:

Theorem 1.1. Let $(\xi, J)$ be a foliated almost contact structure on $\left(V^{4}, \mathcal{F}\right)$. There exists a homotopy $\left\{\left(\xi_{t}, J_{t}\right)\right\} \subset$ $\mathcal{A}(V, \mathcal{F})$ of foliated almost contact structures such that $\left(\xi_{0}, J_{0}\right)=(\xi, J)$ and $\xi_{1}$ is a contact foliation.

The proof of the Theorem also implies a (weak version of the) parametric h-principle:

Corollary 1.2. The maps $\pi_{k} \iota: \pi_{k} \mathcal{C}(V, \mathcal{F}) \longrightarrow \pi_{k} \mathcal{A}(V, \mathcal{F})$ are surjective.

Theorem 1.1 is the case $k=0$ of the parametric result stated in Corollary 1.2. To be precise, Corollary 1.2 is not deduced from the statement of Theorem 1.1 but rather from its method of proof. This is an instance of a flexible phenomenon in foliated contact topology. The argument of Theorem 1.1 certainly uses the classification of overtwisted structures [Eliashberg, 1989], but new ideas coming from foliation theory are needed. In particular, the relative parametric version of a foliated h-principle does not a priori hold (this is implied by Proposition 1.3).

We are also able to present strictly geometric situations, as in the following example. Consider a codimension-1 foliation $\left(N^{3}, \mathcal{F}\right)$ in a closed oriented 3-fold and the circle bundle $\pi: \mathbb{S}(\mathcal{F}) \longrightarrow N$ corresponding to the Euler class $e(T \mathcal{F}) \in H^{2}(N, \mathbb{Z}) \cong[N, B U(1)]$.

Proposition 1.3. The map $\pi_{0} \iota: \pi_{0} \mathcal{C}\left(\mathbb{S}(\mathcal{F}), \pi^{*} \mathcal{F}\right) \rightarrow \pi_{0} \mathcal{A}\left(\mathbb{S}(\mathcal{F}), \pi^{*} \mathcal{F}\right)$ is not injective. 
This Proposition is a direct application of the tight-overtwisted dichotomy in 3-dimensional contact topology. It also turns foliated contact structures into an interesting object from the geometric viewpoint.

Remark 1. There is currently no definition of a flexible-overtwisted-class of foliated contact structures such that, for instance, Theorem 1.1 establishes an isomorphism when restricted to this class. For instance, there are examples of contact foliations with tight and overtwisted leaves (and the existence of a transverse family of overtwisted disks would require tautness of $\mathcal{F}$ ).

Rigidity results in contact topology can be generalized to foliated contact topology. For instance, a foliated Weinstein conjecture can be stated and we may apply the techniques used in the contact case to prove them. In particular, the foliated Weinstein conjecture holds for a particular class of foliated contact structures, confer [del Pino, Presas, 2014].

The article is organized as follows. Section 2 defines the objects of interest. It also contains the foliated counterparts of several classical theorems in contact and symplectic topology. Section 3 describes different procedures for constructing foliated contact (and symplectic) structures. Although Sections 2 and 3 are not entirely required for the proof of Theorem 1.1, they establish the foundations of the theory and provide the reader with intuition on the results and methods that work for these foliated geometries.

Section 4 states the $h$-principles used in the proofs (coming from contact geometry) and technical lemmas which will be used in the proof of Theorem 1.1. Section 5 establishes Theorem 1.1 in the case in which the foliation $\mathcal{F}$ is taut. Section 6 adapts the techniques developed in Section 5 to complete the proof of Theorem 1.1 in the general case and also explains the proof of Corollary 1.2.

\section{Foliated Contact and Symplectic Topology}

Let $V$ be a closed, smooth, oriented manifold. The foliations $\mathcal{F}$ considered on $V$ are smooth, regular, codimension one, cooriented and oriented. The distributions appearing in the article are also assumed to be cooriented and oriented. Given a leaf $\mathcal{L}$ of a foliation $\mathcal{F}$, the natural inclusion of the leaf in the foliation is denoted by $\iota_{\mathcal{L}}: \mathcal{L} \longrightarrow V$.

In this Section we introduce the objects of interest and provide proofs of their basic properties. Subsection 2.1 is dedicated to contact foliations and Subsection 2.2 to symplectic foliations. Constructions and examples shall be explained in Section 3. 


\subsection{Contact foliations}

The central objects in this article are contact foliations. This notion generalizes the concept of a contact fibration and intertwines the contact topology of the leaves with the their global behaviour governed by the foliation.

Definition 2.1. A contact foliation $(\mathcal{F}, \xi)$ on $V$ is a foliation $\mathcal{F}$ on $V$ and a codimension-2 distribution $\xi \subset T \mathcal{F}$ such that $\left(\mathcal{L}, i_{\mathcal{L}}^{*} \xi\right)$ is a contact manifold for every leaf $\mathcal{L}$.

The manifold $V$ must be even dimensional for a contact foliation $(\mathcal{F}, \xi)$ to exist. Given a codimension-2 distribution $\xi \subset T \mathcal{F}$, an extension of $\xi$ is a codimension-1 distribution $\Theta \subset T V$ such that $\xi=\Theta \cap T \mathcal{F}$. The reader should compare this with a contact fibration [Casals, Presas, 2013][Lerman, 1998]. A pair $(\mathcal{F}, \Theta)$ such that $\Theta \cap \mathcal{F}$ is a contact structure on the leaves will be referred to as an extended contact foliation. A pair ( $\beta$, $\alpha)$ of 1 -forms is associated to $(\mathcal{F}, \Theta)$ if $\operatorname{ker} \beta=T \mathcal{F}$ and $\operatorname{ker} \alpha=\Theta$.

Definition 2.2. Let $(\mathcal{F}, \Theta)$ be an extension of a contact foliation $(\mathcal{F}, \xi)$ with associated pair $(\beta, \alpha)$. The associated contact connection is the distribution $\mathcal{H}_{\Theta}=\xi^{\perp d \alpha} \subset(\Theta, d \alpha)$.

The contact connection is a line field contained in $\Theta$ and satisfying $\xi \oplus \mathcal{H}_{\Theta}=\Theta$. In particular, $T \mathcal{F} \oplus \mathcal{H} \Theta=T V$. Observe that $d \alpha$ does not necessarily vanish on $\mathcal{H}_{\Theta}$ : rather, contraction with a vector field in $\mathcal{H}_{\Theta}$ yields a form that vanishes on $\xi$ and $\mathcal{H}_{\Theta}$, so it must be a multiple of $\alpha$. Note that the contact connection does not depend on the choice of $\alpha$, it only depends on the extended distribution $\Theta$. Contact connections have been used before in the case of contact fibrations, see for instance [Casals, Presas, 2013].

Example 2.3. Let $\left(\mathcal{L}, \xi_{\mathcal{L}}=\operatorname{ker} \alpha_{\mathcal{L}}\right)$ be a contact manifold. The manifold $\mathcal{L} \times[0,1]$, with coordinates $(p, t)$, has a natural contact foliation structure given by

$$
\tilde{\mathcal{F}}=\mathcal{L} \times\{t\}, \quad \tilde{\xi}(p, t)=\left(\iota_{\mathcal{L} \times\{t\}}\right)_{*} \xi_{\mathcal{L}}(p)
$$

Consider $\phi \in \operatorname{Cont}\left(\mathcal{L}, \xi_{\mathcal{L}}\right)$ and the associated mapping torus $M(\phi)$ (this is a contact fibration). From the contact foliation viewpoint, it inherits the contact foliation structure $(\mathcal{F}, \xi)$ from $(\mathcal{L} \times[0,1], \tilde{\mathcal{F}}, \tilde{\xi})$ obtained as a quotient. Given a vector field $X \subset T \mathcal{F}$ such that $\phi_{*} X=X$, an extension $(\mathcal{F}, \Theta)$ is obtained by declaring

$$
\Theta=\xi \oplus\left\langle\partial_{t}+X\right\rangle
$$

Denote $H=\alpha_{\mathcal{L}}(X)$. Then the contact connection $\mathcal{H}_{\Theta}$ is the distribution generated by the vector field $\left\langle\partial_{t}+\widetilde{X}\right\rangle$ satisfying the equations

$$
\alpha_{\mathcal{L}}(\tilde{X})-H=0, \quad d \alpha_{\mathcal{L}}(\tilde{X}, v)+d H(v)=0 \quad \forall v \in \operatorname{ker} \alpha_{\mathcal{L}}
$$


Hence $\widetilde{X}$ is the Hamiltonian vector field associated to $H$.

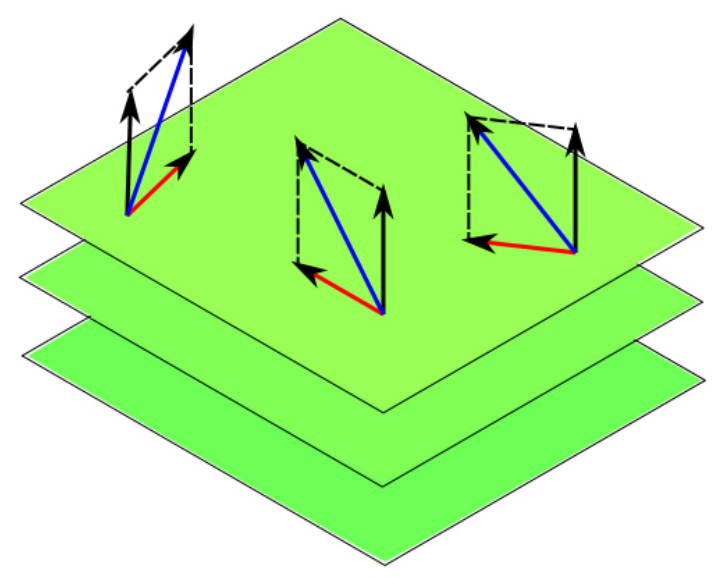

Fig. 1. Foliation $\mathcal{F}$ in green and the vector fields $\partial_{t}$ (black), $X$ (red), and $\partial_{t}+X$ (blue).

Given an extension $(\mathcal{F}, \Theta)$ defined by $(\beta, \alpha)$, we associate two vector fields $T$ and $R$ uniquely determined by the following equations:

$$
\begin{gathered}
\alpha(T)=0, \quad\left(i_{T} d \alpha\right) \wedge \alpha=0, \quad \beta(T)=1 \\
\alpha(R)=1, \quad\left(i_{R} d \alpha\right) \wedge \beta=0, \quad \beta(R)=0 .
\end{gathered}
$$

These vector fields $T$ and $R$ will be referred to as the transverse field and the Reeb field of $(\beta, \alpha)$. Note that $\mathcal{H}_{\Theta}=\langle T\rangle$.

The parallel transport between the leaves in Example 2.3 is by contactomorphisms. This is a particular instance of the following

Lemma 2.4. Let $(\mathcal{F}, \Theta)$ be an extended contact foliation and $(\beta, \alpha)$ an associated pair. Then

$$
\mathcal{L}_{T} \alpha=d \alpha(T, R) \alpha
$$

In particular the distribution $\Theta$ is preserved by the flow of the transverse field $T$ of $(\beta, \alpha)$.

It does not hold in general that $T$ preserves $\mathcal{F}$ since that would imply $d \beta=0$.

Proof. The Cartan formula gives $\mathcal{L}_{T} \alpha=d i_{T} \alpha+i_{T} d \alpha=i_{T} d \alpha$. The statement follows from the fact that $i_{T} d \alpha=d \alpha(T, R) \alpha$, which can be readily verified by evaluation in each factor of $\xi \oplus R \oplus T$.

Consider the space $\mathcal{E}(\mathcal{F}, \xi)=\{(\mathcal{F}, \Theta):(\mathcal{F}, \Theta)$ is an extension of $(\mathcal{F}, \xi)\}$. The space of connection 1-forms in a smooth vector bundle has a natural affine structure. Similarly, one can prove the following: 
Lemma 2.5. $\mathcal{E}(\mathcal{F}, \xi)$ has an affine structure.

Proof. Consider an extension $\left(\mathcal{F}, \Theta_{0}\right)$ with associated pair $\left(\beta_{0}, \alpha_{0}\right)$. The space

$$
A=\left\{\alpha \in \Omega^{1}(V): \alpha \wedge \beta_{0}=\alpha_{0} \wedge \beta_{0}\right\}
$$

is an affine space modelled on the space of 1 -forms vanishing on $\mathcal{F}$. The map $\Psi: A \longrightarrow \mathcal{E}(\mathcal{F}, \xi)$ defined by $\Psi(\alpha)=(\mathcal{F}, \operatorname{ker} \alpha)$ is bijective and endows $\mathcal{E}(\mathcal{F}, \xi)$ with an affine structure.

In particular, the space $\mathcal{E}(\mathcal{F}, \xi)$ is contractible and the choice of extension for a foliated contact structure is unique up to homotopy.

The previous definitions and facts suffice for the reader to follow the proof of Theorem 1.1. However, we consider it appropriate to include a short discussion on the transformations appearing in foliated contact topology. A diffeomorphism of $V$ that preserves both $\mathcal{F}$ and $\xi$ will be called a foliated contactomorphism. The infinitesimal symmetries are described as follows.

Definition 2.6. Let $(V, \mathcal{F}, \xi)$ be a contact foliation. The space of contact vector fields of $(V, \mathcal{F}, \xi)$ is defined as the subspace of $\mathfrak{X}(V)$ of those vector fields that preserve $\mathcal{F}$ and $\xi$.

If an extension $(\mathcal{F}, \Theta)$ is fixed and an associated pair $(\beta, \alpha)$ is given, the space of contact vector fields is equivalently defined as:

$$
\begin{array}{rlr}
\mathcal{C}(\xi)=\{X \in \mathfrak{X}(V): & \mathcal{L}_{X}(\alpha \wedge \beta)=f \alpha \wedge \beta & \text { for some } f \in C^{\infty}(V) \\
\mathcal{L}_{X} \beta=g \beta & \text { for some } \left.g \in C^{\infty}(V)\right\} .
\end{array}
$$

A flow by foliated contactomorphisms is induced by a 1-parametric family of contact vector fields.

Lemma 2.7. The space $\mathcal{C}(\xi)$ is a Lie algebra. It contains a distinguished ideal $\mathcal{C}(\xi) \cap \mathfrak{X}(\mathcal{F})$.

Proof. The first statement follows since the space of vector fields preserving a distribution is a Lie algebra. The second claim is immediate, since $[X, Y] \in T \mathcal{F}$, for $X$ preserving $\mathcal{F}$ and $Y \in \mathcal{F}$.

This incursion into foliated contact vector fields and the use of extensions of a contact foliation lead to a proof of the foliated version of Gray's stability in contact topology (i.e. the moduli space of contact structures is discrete). The precise statement reads as follows. 
Lemma 2.8 (Foliated Gray's Stability). Let $\mathcal{F}$ be a codimension 1-foliation on a closed manifold $V$ and consider a family $\left\{\xi_{t}\right\}_{t \in[0,1]}$ of codimension-2 distributions such that $\left(\mathcal{F}, \xi_{t}\right)$ is a foliated contact structure for every $t \in[0,1]$. Then there exists a global flow $\left\{\phi_{t}\right\}_{t \in[0,1]} \in \operatorname{Diff}(V)$ tangent to $\mathcal{F}$ such that $\phi_{t}^{*} \xi_{t}=\xi_{0}$.

Proof. We consider a smooth 1-parametric family of extensions $\Theta_{t}$ and their associated pairs $\left(\beta, \alpha_{t}\right)$. We require a flow $\phi_{t}$ tangent to the leaves (and therefore preserving $\mathcal{F}$ ) such that $\phi_{t}^{*} \xi_{t}=\xi_{0}$. In terms of the associated forms this reads as

$$
\phi_{t}^{*}\left(\alpha_{t}\right) \wedge \beta=g_{t} \alpha_{0} \wedge \beta
$$

for a suitable choice of $\left\{g_{t}\right\}_{t} \in C^{\infty}(V)$, since $\phi_{t}$ preserves $\beta$ up to scaling. We now apply the foliated version of Moser's argument.

Denote by $X_{t}$ the vector field generating the required flow $\phi_{t}$ (that is, $X_{t} \circ \phi_{t}=\dot{\phi}_{t}$ ) and we further suppose that $X_{t}$ is contained in the contact structures $\xi_{t}$. Differentiating the above condition with respect to $t$ we obtain:

$$
\phi_{t}^{*}\left(\mathcal{L}_{X_{t}} \alpha_{t}+\dot{\alpha}_{t}\right) \wedge \beta=g_{t}^{\prime} \alpha_{0} \wedge \beta=\frac{g_{t}^{\prime}}{g_{t}}\left(\phi_{t}^{*} \alpha_{t}\right) \wedge \beta .
$$

Define $\lambda_{t}=\left(\phi_{t}\right)_{*} \frac{g_{t}^{\prime}}{g_{t}}$ and $\left(\phi_{t}\right)_{*} \beta=m_{t} \beta$. Equation (1) implies

$$
\left(\mathcal{L}_{X_{t}} \alpha_{t}+\dot{\alpha}_{t}\right) \wedge\left(m_{t} \beta\right)=\lambda_{t} m_{t} \alpha_{t} \wedge \beta
$$

Since $m_{t}$ is strictly positive, the equation can we written as

$$
\begin{aligned}
& \left(\mathcal{L}_{X_{t}} \alpha_{t}+\dot{\alpha}_{t}\right) \wedge \beta=\lambda_{t} \alpha_{t} \wedge \beta, \\
& \left(i_{X_{t}} d \alpha_{t}+\dot{\alpha}_{t}\right) \wedge \beta=\lambda_{t} \alpha_{t} \wedge \beta .
\end{aligned}
$$

This is an equation in 1-forms. In particular, it has to be satisfied by the Reeb vector $R_{t}$, thus yielding the condition

$$
\left(i_{R_{t}} \dot{\alpha}_{t}\right) \beta=\lambda_{t} \beta
$$

This reads $\phi_{t}^{*}\left(i_{R_{t}} \dot{\alpha}_{t}\right)=\left(\ln g_{t}\right)^{\prime}$, which is an ODE with an unique solution once the initial condition $g_{0}=1$ is fixed. Now, since $R_{t} \in \operatorname{ker}\left(\dot{\alpha}_{t}-\lambda_{t} \alpha_{t}\right)$, Equation (2) can solved uniquely for $X_{t} \in \xi_{t}$.

There are foliated analogues of several concepts and results in contact topology. We have introduced the essential notions, and the reader should be able to define any further objects and prove foliated versions of basic results. 
See for instance Lemmas 2.13 and 2.15 .

\subsection{Symplectic foliations}

In this subsection we treat the case of (strong) symplectic foliations. It is not required in order to prove Theorem 1.1, however it serves as a foundational subsection and contributes to a better understanding of foliated contact topology.

We begin with the simpler notion of a symplectic foliation.

Definition 2.9. Let $\mathcal{F}$ be a codimension-1 foliation on a smooth manifold $M$ and $\omega \in \Omega^{2}(\mathcal{F})=\Lambda^{2}\left(T^{*} \mathcal{F}\right)$ a 2 -form. The pair $(\mathcal{F}, \omega)$ is a symplectic foliation if, for every leaf $\mathcal{L},\left(\mathcal{L}, i_{\mathcal{L}}^{*} \omega\right)$ is a symplectic manifold.

These objects are a generalisation of symplectic fibrations, see [McDuff, Salamon, 1998], and they are also referred to as regular Poisson structures, confer [Hector, 1992]. The subtlety in the symplectic case, in contrast to the contact case, is the closedness of the possible extensions of the 2-form $\omega$. Given a symplectic foliation, we can consider the space of closed 2 -forms $\Omega$ such that $\left.\Omega\right|_{T \mathcal{F}}=\omega$. Then, a pair $(\mathcal{F}, \Omega)$ with such an $\Omega$ will be called an extension of $(\mathcal{F}, \omega)$. Symplectic foliations with a fixed extension are also referred to as 2-calibrated structures [Ibort, Martinez Torres, 2004].

Consider the space $\mathfrak{E}(\mathcal{F}, \omega)$ of all extensions of $\omega$. In order to distinguish the cases in which an extension exists we introduce the following notation:

Definition 2.10. A symplectic foliation $(\mathcal{F}, \omega)$ is called a strong symplectic foliation, or simply a s-symplectic foliation, if the space $\mathfrak{E}(\mathcal{F}, \omega)$ is non-empty.

The symplectic version of Lemma 2.5 also holds.

Lemma 2.11. $\mathfrak{E}(\mathcal{F}, \omega)$ is either empty or has a natural affine structure space.

Proof. Suppose that $\mathfrak{E}(\mathcal{F}, \omega)$ is non-empty, fix an extension $\Omega_{0}$, and let $\beta$ be a defining 1 -form for $\mathcal{F}$. Then the set

$$
\left\{\Omega \in \Omega^{2}(M): \Omega \wedge \beta=\Omega_{0} \wedge \beta, d \Omega=0\right\}
$$

is an affine space modelled on the space of closed 2-forms that vanish along the foliation.

There exist cohomological obstructions for a symplectic foliation to be strong symplectic. See [Crainic, Fernandes, 2004, Example 10] and [Vaisman, 1994]. The assumption of a foliation being $s$-symplectic is meaningful in the sense that many constructions from symplectic topology are likely to extend to the foliated setting only 
under this hypothesis. Approximately holomorphic techniques, see [Martinez Torres, 2013], have been shown to work and they offer a fruitful approach for understanding the leaf space of $s$-symplectic foliations. Additionally, in $s$-symplectic foliations given by a closed $\beta$, being particular instances of stable hamiltonian structures, it is to be expected for Floer techniques to translate nicely.

The closedness of the extension 2-form is relevant in regard to the concept of a symplectic connection.

Definition 2.12. Let $(\mathcal{F}, \omega)$ be a symplectic foliation. Given an extension $(\mathcal{F}, \Omega)$, the symplectic connection is the distribution $\mathcal{H}_{\Omega}=(T \mathcal{F})^{\perp \Omega}$.

If a defining form $\beta$ for the foliation is chosen, a symplectic connection determines a distinguished transverse vector field $T$ by

$$
\Omega(T)=0, \quad \beta(T)=1
$$

Just as closedness of the extension is the condition required in symplectic fibrations for the parallel transport to be by symplectomorphisms, see [McDuff, Salamon, 1998, Lemma 6.18], closedness of $\Omega$ implies that $T$ preserves $\Omega:$

$$
\mathcal{L}_{T} \Omega=d i_{T} \Omega+i_{T} d \Omega=0
$$

The vector field $T$ has been known in Poisson geometry for a while [Vaisman, 1994, Definition 4.8]. For strong symplectic foliations defined by a closed 1-form it was proven in [Guillemin, Miranda, Pires, 2011] that $T$ preserves the Poisson structure. From our perspective, this can be rephrased by saying that, $\beta$ being closed, $T$ not only preserves $\Omega$, but also $\mathcal{F}$. A particularly simple case is when $[\beta] \in H^{1}(M)$ is rational: then $(M, \mathcal{F}, \omega)$ is actually the mapping torus of a symplectomorphism, and $\Omega$ can be assumed to be closed.

We do not need to introduce the notion of Hamiltonian vector fields for a symplectic foliation, since it will not be used in the proofs. The interested reader may use Rybicki [2001]as an useful reference.

Let us establish Moser's Lemma and Darboux's Theorem for the case of foliated symplectic topology. Their proofs are left to the reader.

Lemma 2.13 (Moser Stability, see [Hector, Macias, Saralegi, 1989]). Consider a foliation $\mathcal{F}$ on a closed manifold $M$ and $\left\{\omega_{t}\right\}_{t \in[0,1]}$ a smooth family of foliated 2-forms such that $\left(\mathcal{F}, \omega_{t}\right)$ is a symplectic foliation for every $t$. Let $\left\{\Omega_{t}\right\}$ be a smooth family of associated extensions. Suppose that $\left[\Omega_{t}\right] \in H_{D R}^{2}(M)$ is constant, then there exists a global flow $\left\{\phi_{t}\right\}_{t} \in \operatorname{Diff}(M)$ tangent to $\mathcal{F}$ and such that $\phi_{t}^{*} \omega_{t}=\omega_{0}$.

Darboux's Theorem shall provide a local normal form for a symplectic foliation. We describe this local model in the following example. 
Example 2.14. The product manifold $\mathbb{R}(t) \times \mathbb{C}^{n}(x, y)$ - by which we mean that $t$ is the coordinate in $\mathbb{R}$ and $(x, y)=\left(x_{1}, \cdots, x_{n}, y_{1}, \cdots, y_{n}\right)$ the coordinates in $\mathbb{C}^{n}-$ can be endowed with the strong symplectic foliation $\left(\mathcal{F}_{s t}, \Omega_{s t}\right)$ defined by

$$
\mathcal{F}_{s t}=\coprod_{t \in \mathbb{R}}\{t\} \times \mathbb{C}^{n}, \quad \Omega_{s t}=\sum_{i=1}^{n} d x_{i} \wedge d y_{i} .
$$

The standard defining form for $\mathcal{F}_{s t}$ is the 1 -form $\beta_{s t}=d t$.

Lemma 2.15 ((Strict) Darboux's Theorem). Consider a symplectic foliation $(\mathcal{F}, \omega)$ on $M$ and a point $p \in M$. Then there exist a small $\varepsilon>0$ and an embedding

$$
\begin{gathered}
\phi: D_{\varepsilon}^{2 n} \times(-\varepsilon, \varepsilon) \longrightarrow M \\
\phi(0,0)=p, \quad \phi^{*} \mathcal{F}=\mathcal{F}_{s t}, \quad \phi^{*} \omega=\left.\Omega_{s t}\right|_{\mathcal{F}_{s t}} .
\end{gathered}
$$

If $(\mathcal{F}, \omega)$ is actually $s$-symplectic with extension $\Omega$, then it can further be achieved that $\phi^{*} \Omega=\Omega_{s t}$.

Darboux's Theorem also holds for contact foliations (the proof relies on the foliated Gray's stability theorem). The details are left to the interested reader.

This Section 2 has introduced the basic definitions and properties of contact and (strong) symplectic foliations. Section 3 provides some constructions and examples which illustrate the richness of these geometries.

\section{Constructions and Tautness}

In this section we present possible constructions of foliated contact and symplectic structures. These are natural generalizations of methods used in contact and symplectic topology, although the structure of the foliation provides interesting classes of examples. These subsections can be read independently.

Subsection 3.1 briefly recalls the definition of a taut foliation (which will be used in Sections 4 and 5). An interesting example is presented in Subsection 3.2. Subsection 3.3 introduces the space of foliated contact elements and proves Proposition 1.3. Finally, Subsections 3.4, 3.5 and 3.6 explain contactization, symplectization and foliated connected sum along divisors.

\subsection{Tautness}

This is a property associated to a codimension one foliation $\mathcal{F}$ on a smooth $(n+1)$-fold $V$. A foliation $\mathcal{F}$ on a smooth manifold $V$ is said to be (topologically) taut if for every leaf there exists a transverse circle intersecting that leaf. 
There is also the notion of a geometrically taut foliation: $\mathcal{F}$ is geometrically taut if there exists a closed form $\tau \in \Omega^{n}(V)$ such that $i_{\mathcal{L}}^{*} \tau$ is a volume form for every leaf $\mathcal{L}$. This is equivalent to the existence of a complete non-vanishing vector field $X \in \mathfrak{X}(V)$ transverse to $\mathcal{F}$ and preserving some volume form $\nu \in \Omega^{n+1}(V)$ (i.e. $\mathcal{L}_{X} \nu=0$ ). A taut foliation $\mathcal{F}$ is geometrically taut, and the converse holds if the ambient manifold $V$ is closed, see [Sullivan, 1976, Theorem II.20].

A $s$-symplectic foliation $(M, \mathcal{F}, \omega)$ is geometrically taut, since for any choice of extension $\Omega$, we have that the closed $(2 n)$-form $\Omega^{n}$ is a leafwise volume form. Note that geometrically taut foliations and $s$-symplectic foliations are the same objects when $n=1$ and, as such, $s$-symplectic foliations have been considered as a natural generalisation of the former, see [Martinez Torres, 2006]. In contrast to this, a contact foliation $(M, \mathcal{F}, \xi)$ does not come for free with a closed $(2 n+1)$-form inducing a volume on the leaves: it is easy to check that for any extension $\Theta$ with defining form $\alpha$, the natural leafwise volume form $\alpha \wedge(d \alpha)^{n}$ is not closed necessarily.

Theorem 1.1 makes this distinction between contact and $s$-symplectic foliations clear, since it states that any foliation, taut or not, admits a contact foliation. As a motivating example, in Subsection 3.3 we will construct a family of non-taut foliations endowed with foliated contact structures.

Theorem 1.1 will first be proven for taut foliations in Section 5 .

\subsection{An example}

Let us construct a foliated contact structure on a codimension one foliation on the 4 -torus $\mathbb{T}^{4}$. There are four natural types of foliations on the 4-torus obtained by quotienting the horizontal foliation of a 4-polydisk by 3-polydisks. In coordinates $\mathbb{T}^{4}(t, x, y, z)$ their defining equations have the form

$$
\beta=(p, q, r, s) \cdot(d x, d y, d z,-d t), \quad(p, q, r, s) \in \mathbb{R}^{4} .
$$

The numbers $(p, q, r, s)$ generate a $\mathbb{Q}$-submodule $A$ of $\mathbb{R}$. The leaves are diffeomorphic to $\left(\mathbb{S}^{1}\right)^{(4-\operatorname{rank}(A))} \times \mathbb{R}^{\operatorname{rank}(A)-1}$.

Let us endow such foliations with a foliated contact structure. Suppose that $s=1$ and consider the form

$$
\alpha=\sin (2 \pi z) d x+\cos (2 \pi z) d y .
$$


This is a well--defined 1 -form on $\mathbb{T}^{4}$. The condition for a foliated contact structure reads

$$
\begin{aligned}
\alpha \wedge d \alpha & \wedge \beta=(\sin (2 \pi z) d x+\cos (2 \pi z) d y) \wedge(2 \pi \cos (2 \pi z) d z \wedge d x-2 \pi \sin (2 \pi z) d z \wedge d y) \wedge \beta= \\
= & (2 \pi d x \wedge d y \wedge d z) \wedge(p \cdot d x+q \cdot d y+r \cdot d z-d t)=2 \pi \cdot d t \wedge d x \wedge d y \wedge d z>0
\end{aligned}
$$

Hence $\alpha$ defines a foliated contact structure for any 1 -form $\beta$ as above. In particular, we obtain a contact foliation with (dense) tight contact $\mathbb{R}^{3}$ leaves on $\mathbb{T}^{4}$.

This example is of a particular interest regarding a Weinstein-type conjecture in foliated contact topology. In this last irrational case, the foliated Reeb vector field has no periodic orbits (even though the ambient manifold $\mathbb{T}^{4}$ is compact). This example must be placed in contrast to the case with overtwisted leaves, see [del Pino, Presas, 2014].

\subsection{Foliated contact elements}

Consider a pair $(W, \mathcal{F})$ given by a codimension one, cooriented foliation $\mathcal{F}$ on $W$ and the manifold $V=\mathbb{P}\left(T^{*} \mathcal{F}\right)$, the projectivised cotangent bundle of $\mathcal{F}$. Since $\pi: V \longrightarrow W$ is a fibre bundle, the foliation $\mathcal{F}$ can be pull-backed to $V$ to a foliation $\mathcal{F}_{V}=\pi^{*} \mathcal{F}$. The projectivization of the cotangent bundle of a manifold has a canonical contact structure. Similarly, the foliated manifold $V$ has a natural foliated contact structure defined by

$$
\left(\xi_{V}\right)_{p}=\left\{v \in T_{p} \mathcal{F}_{V}: p\left(\pi_{*} v\right)=0\right\}, \quad p \in V
$$

where the point $p$ is identified with a 1 -form in $T_{\pi(p)} \mathcal{F}$ (which is well-defined up to scaling).

In particular, the foliated contact structure restricted to a leaf coincides with the space of contact elements of the leaf. In the same vein, the sphere bundle $S=\mathbb{S}\left(T^{*} \mathcal{F}\right)$ associated to the cotangent space of the foliation is a foliated contact manifold $\left(S, \mathcal{F}_{S}, \xi_{S}\right)$ that restricts to the space of cooriented contact elements over each leaf. The foliated contact structure $\left(\mathcal{F}_{S}, \xi_{S}\right)$ can also be obtained via the pullback of $\left(\mathcal{F}_{V}, \xi_{V}\right)$ through the double-cover $S \longrightarrow V$.

Let us provide an example of two non-isotopic foliated contact structures which are homotopic as foliated almost contact structures.

Proof of Proposition 1.3. The standard foliated contact structure on the space of foliated contact elements has tight leaves. This is standard in 3-dimensional contact topology: the space of cooriented contact elements of a 
(possibly open) surface is tight. The proof of Theorem 1.1 shall imply that a foliated contact structure with (at least) an overtwisted leaf can also be constructed in the same homotopy class of foliated almost contact structures. Those two structures cannot be homotopic as foliated contact structures, because Lemma 2.8 would imply isotopy of the two contact structures restricted to any leaf.

A particular example we consider clarifying is constructed in the following proposition.

Proposition 3.1. Consider the Reeb foliation $\left(\mathbb{S}^{3}, \mathcal{F}\right)$ and its associated space of foliated contact elements $\left(\mathbb{S}^{3} \times \mathbb{S}^{1}, \mathcal{F}_{c}, \xi_{c}\right)$. There exists a homotopy of the almost contact structures that produces a contact foliation with tight and overtwisted leaves.

Proof. Fix a loop $\gamma: \mathbb{S}^{1} \rightarrow \mathbb{S}^{3}$ transverse to the foliation $\mathcal{F}$ (and thus avoiding the unique torus leaf). Its lift to the space of foliated contact elements is a transverse loop of the form

$$
\begin{aligned}
\widetilde{\gamma}: \mathbb{S}^{1} & \longrightarrow \mathbb{S}^{3} \times \mathbb{S}^{1} \\
\theta & \longmapsto(\gamma(\theta), 1)
\end{aligned}
$$

Let us insert a family of Lutz twists (refer to Section 6 for a more detailed account of this construction) by considering an embedding

$$
\Gamma: \mathbb{S}^{1} \times \mathbb{S}^{1} \longrightarrow \mathbb{S}^{3} \times \mathbb{S}^{1}
$$

such that $\Gamma(\theta, 1)=\widetilde{\gamma}(\theta)$ and the $\kappa$-curve $\{s \longmapsto \kappa(s)=\Gamma(\theta, s)\}$ is tangent to $\mathcal{F}_{c}$ and transverse to $\xi_{c}$.

Then we perform a 1 -parametric family of Lutz twists along the $\theta$-family of $\kappa$-curves. The resulting contact structure certainly has overtwisted leaves. However, the leaf $T^{2} \times \mathbb{S}^{1}$ corresponding to the lift of the unique compact leaf on $\mathcal{F}$ is tight.

Note that the same proof works for any foliation $\left(M^{3}, \mathcal{F}\right)$ with a Reeb component: the space of foliated contact elements associated to $\left(M^{3}, \mathcal{F}\right)$ possesses a foliated contact structure with tight and overtwisted leaves.

\subsection{Contactization}

Let $(M, \lambda)$ be an exact symplectic manifold, the contactization $\mathcal{C}(M, \lambda)$ of $(M, \lambda)$ is the manifold $M \times \mathbb{R}(t)$ endowed with the contact structure $\xi=\operatorname{ker}(\lambda-d t)$.

Definition 3.2. Let $(M, \mathcal{F}, \omega)$ be an $s$-symplectic manifold admitting an exact extension $\Omega=d \lambda$. Then $(M \times \mathbb{R}(t), \mathcal{F} \times \mathbb{R}, \operatorname{ker}(\lambda-d t))$ is called the contactization $\mathcal{C}(M, \mathcal{F}, \omega)$ of $(M, \mathcal{F}, \omega)$. 
In case the symplectic manifold $(M, \omega)$ is not exact there also exists a contactization (known as prequantization). Let $(\mathcal{F}, \omega)$ be an $s$-symplectic foliation on $M$, with an extension $\Omega$ such that $[\Omega /(2 \pi)]$ is integral and consider the principal circle bundle $L_{\Omega} \longrightarrow M$ associated to $\Omega$. Construct a connection 1-form $\alpha \in \Omega^{1}\left(L_{\Omega}\right)$ with curvature $\Omega$.

Definition 3.3. The foliated contact manifold $\left(L_{\Omega}, \pi^{*} \mathcal{F}, \operatorname{ker} \alpha\right)$ is said to be the Boothby-Wang contact foliation associated to the s-symplectic foliation $(M, \mathcal{F}, \omega)$ with extension $\Omega$.

Note that the Boothby-Wang contact foliation over a closed base is taut because the $s$-symplectic foliation is taut. In [Alcalde-Cuesta, 1993], an obstruction for a symplectic foliation to be strong was formulated in terms of the existence of a fibre bundle with curvature form given by a closed extension. Moreover, a more general discussion of affine connections in Poisson geometry can be found in [Fernandes, 2000].

\subsection{Symplectization}

Let $(V, \xi=\operatorname{ker} \alpha)$ a contact manifold. The symplectic manifold $\left(V \times \mathbb{R}(t), d\left(e^{t} \alpha\right)\right)$ is known as the symplectization of $(V, \xi)$.

Definition 3.4. Given an extended contact foliation $(V, \mathcal{F}, \xi, \Theta)$ with associated pair $(\beta, \alpha)$, the symplectization of $(V, \mathcal{F}, \xi, \alpha)$ is the $s$-symplectic foliation

$$
\left(V \times \mathbb{R}(t), \mathcal{F} \times \mathbb{R}, \omega=\left.d\left(e^{t} \alpha\right)\right|_{\mathcal{F}}\right) .
$$

Notice that $\Omega=d\left(e^{t} \alpha\right)$ is an extension of $\omega$ (and thus $\Omega$ is exact). The $s$-symplectic foliation obtained in the construction does not depend on the particular choice of $\alpha$ and $\Theta$ (up to foliated symplectomorphism).

Observe that the symplectization is geometrically taut, since it is a $s$-symplectic foliation. However it is not necessarily taut; if this were the case the starting contact foliation would be taut.

\subsection{Foliated contact divisor connected sum}

Consider a contact foliation $(V, \mathcal{F}, \xi)$ on a $(2 n+2)$-fold $V$ and let $S$ be a $2 n$-dimensional submanifold transverse to $\mathcal{F}$ and $\xi$. Then $S$ inherits a codimension-1 foliation $\mathcal{F}_{S}$ with foliated contact structure $\xi_{S}=\xi \cap T \mathcal{F}_{S}$. We say that $\left(S, \mathcal{F}_{S}, \xi_{S}\right)$ is a foliated contact divisor. Generalizing [Geiges, 2008, Theorem 2.5.15], Lemma 2.8 implies that the tubular neighbourhood of $S$ is uniquely determined by the conformal symplectic structure of its normal bundle. Since its normal bundle is a 2-dimensional disc bundle, the foliated contact structure of its tubular 
neighbourhood depends only on its oriented topological type.

Suppose that the normal bundle of $S$ is trivial. Then, there is a diffeomorphism $\phi: S \times D_{2 \varepsilon}^{2} \rightarrow \operatorname{Op}(S), \varepsilon>0$, where $\mathrm{Op}(S) \subset V$ denotes a small tubular neighbourhood of $S$. Let $\left(\alpha_{S}, \beta_{S}\right)$ be an associated pair for $\left(S, \mathcal{F}_{S}, \xi_{S}\right)$. Then the pullback of the contact foliation by the embedding $\phi$ can be chosen to be:

$$
\left(S \times D_{2 \varepsilon}^{2}, \mathcal{F}_{S} \times D_{2 \varepsilon}^{2}, \operatorname{ker}\left(\alpha_{\mathrm{Op}(S)}\right)\right) \text { with associated pair }\left(\beta_{\mathrm{Op}(S)}, \alpha_{\mathrm{Op}(S)}\right)=\left(\phi^{*} \beta_{S}, \phi^{*} \alpha_{S}+r^{2} d \theta\right) .
$$

This is the local model along the foliated contact divisor. We describe the framework in which we can perform a foliated contact connected sum along such a foliated contact divisor.

Let $\left(V_{0}, \mathcal{F}_{0}, \xi_{0}\right)$ and $\left(V_{1}, \mathcal{F}_{1}, \xi_{1}\right)$ be two contact foliations and $f_{0}: S \longrightarrow V_{0}, f_{1}: S \longrightarrow V_{1}$ two embeddings of $S$ as a foliated contact divisor with trivial normal bundle. There exist two neighbourhoods $\operatorname{Op}\left(S, V_{0}\right)$ and $\operatorname{Op}\left(S, V_{1}\right)$ and two embeddings

$$
f_{0}: S \times D_{2 \varepsilon}^{2} \longrightarrow \mathrm{Op}\left(S, V_{0}\right), \quad f_{1}: S \times D_{2 \varepsilon}^{2} \longrightarrow \mathrm{Op}\left(S, V_{1}\right)
$$

conforming to the local model described above (and extending $f_{0}$ and $f_{1}$ ).

The gluing region is the open manifold $\mathcal{S}=S \times\left(-\varepsilon^{2}, \varepsilon^{2}\right) \times \mathbb{S}^{1}$ contact foliated as

$$
\left(\mathcal{S}, \mathcal{F}_{S} \times\left(-\varepsilon^{2}, \varepsilon^{2}\right) \times \mathbb{S}^{1}, \operatorname{ker}\left(\alpha_{\mathcal{S}}\right)\right) \text { with associated pair }\left(\beta_{\mathcal{S}}, \alpha_{\mathcal{S}}\right)=\left(\beta_{S}, \alpha_{S}+t d \theta\right)
$$

Note the linearity in the $t$-coordinate. Define the maps

$$
\begin{array}{r}
F_{0}: S \times\left(0, \varepsilon^{2}\right) \times \mathbb{S}^{1} \longrightarrow \mathrm{Op}\left(S, V_{0}\right), \quad(p, t, \theta) \\
\left.F_{1}: S \times\left(-\varepsilon^{2}, 0\right) \times \mathbb{S}^{1} \longrightarrow \mathrm{Op}\left(S, V_{1}\right), \quad(p, t, \theta) \longmapsto t_{1}, \theta\right)
\end{array}
$$

Then the topological connected sum

$$
V_{0} \#_{S} V_{1}=\left(V_{0} \backslash f_{0}(S)\right) \cup_{F_{0}} \mathcal{S} \cup_{F_{1}}\left(V_{1} \backslash f_{1}(S)\right)
$$

with the foliated contact models introduced above inherits a foliated contact structure. The related construction for symplectic foliations is discussed in [Ibort, Martinez Torres, 2004], though it does not preserve strongness. 
Sections 2 and 3 have presented the definitions, results and constructions in foliated contact and symplectic topology. The remaining Sections 4, 5 and 6 shall focus on the proof of Theorem 1.1.

\section{4 h-Principle and Local Models}

In this Section we state the $\mathrm{h}$-principles and the technical lemmas involved in the proof of Theorem 1.1. Subsections 4.1 and 4.2 introduce the appropriate version of the $\mathrm{h}$-principles for the open and the closed case respectively. Subsection 4.3 contains the topological local models and Subsection 4.4 begins to construct the foliated contact structure on the 3-skeleton of $\left(V^{4}, \mathcal{F}\right)$.

For any subset $A, \operatorname{Op}(A)$ will denote an open neighbourhood of $A$. It is not any fixed open neighbourhood, but rather a neighbourhood as small as necessary. The notation $M(p)$ will be used to denote the manifold $M$ with coordinates $(p)$.

\section{1 h-Principle for open manifolds}

The $\mathrm{h}$-principles proven by M. Gromov [Gromov, 1986] include a parametric (and relative) $\mathrm{h}$-principle for the existence of contact structures on open manifolds. This h-principle is also proven in [Eliashberg, Mishachev, 2002]. Let us state the precise result we use in the proof of Theorem 1.1.

Theorem 4.1. ([Eliashberg, Mishachev, 2002, 10.3.2][Gromov, 1986]) Let $V$ be a smooth manifold. Let $U \subset V$ be an open submanifold and $A \subset U$ a CW-complex of codimension at least 2. Let $K$ be a compact space and $L \subset K$ a closed subset. Consider a continuous family $\left\{\left(\xi_{t}, J_{t}\right)\right\}_{t \in K}$ of almost contact structures on $V$ which are contact in $U$ for $t \in L$ and are contact in $\operatorname{Op}(A)$ for $t \in K$.

Then, there exists a continuous deformation $\left\{\left(\xi_{t, s}, J_{t, s}\right)\right\}_{s \in[0,1]}$, relative to $U \times L \cup A \times K$ and supported in $\operatorname{Op}(U)$, such that $\left\{\left(\xi_{t, 1}, J_{t, 1}\right)\right\}_{t \in K}$ is a family of contact structures on $U$.

Observe that the dependence on the parameter can be supposed to be smooth since the condition for a contact structure is open (and thus preserved by small smoothing perturbations). Theorem 4.1 allows us to construct a foliated contact structure in a neighborhood of the 3-skeleton. This is explained in Subsection 4.4.

\subsection{Classification of overtwisted contact structures}

There also exists a subclass of closed contact manifolds satisfying an existence $\mathrm{h}$-principle. This is the main result in [Eliashberg, 1989]. Let us define this class. The disk

$$
\{z=0, \rho \leq \pi\} \subset\left(\mathbb{R}^{3}(z, r, \theta), \xi_{\text {ot }}=\operatorname{ker}\{\cos (r) d z+r \sin (r) d \theta\}\right)
$$


with the germ of the contact structure $\xi_{o t}$ is called the standard overtwisted disk. An almost contact 3-fold $\left(V^{3}, \xi\right)$ is said to be overtwisted if there exists an embedded disk $D$ such that $(\operatorname{Op}(D), \xi)$ is contactomorphic to the standard overtwisted disk. The class of overtwisted (almost) contact manifolds is flexible, i.e. it is classified by its homotopy data. The corresponding h-principle can be stated as follows.

Theorem 4.2. ([Eliashberg, 1989, Theorem 3.1.1]) Let $V$ be a smooth 3 -fold. Let $A \subset V$ be a CW-complex such that $V \backslash A$ is connected. Let $K$ be a compact space and $L \subset K$ a closed subset. Fix a disc $\Delta \subset V \backslash A$. Consider a continuous family $\left\{\left(\xi_{t}, J_{t}\right)\right\}_{t \in K}$ of almost contact structures on $V$ which are contact in $V$ for $t \in L$, contact in $\operatorname{Op}(A) \cup \operatorname{Op}(\Delta)$ for $t \in K$ and have $\Delta$ as an overtwisted disc for all $t \in K$.

Then, there exists a continuous deformation $\left\{\left(\xi_{t, s}, J_{t, s}\right)\right\}_{s \in[0,1]}$, relative to $V \times L \cup \mathrm{Op}(A) \times K$ such that $\left\{\left(\xi_{t, 1}, J_{t, 1}\right)\right\}_{t \in K}$ is a family of contact structures on $V$.

Theorem 4.2 is used in Sections 5 and 6 in order to extend the foliated contact structure on a neighborhood of the 3-skeleton to the interior of the 4-simplices.

\subsection{Topological local models}

Consider a pair $\left(V^{4}, \mathcal{F}^{3}\right)$. A smooth simplex $\sigma: \Delta \longrightarrow V$ is said to be linear with respect to $\mathcal{F}$ if its image is contained in the image of a trivializing foliation chart for $\mathcal{F}$ and $\mathcal{F}$ is transverse to all its faces. By face we mean a subsimplex of any dimension. In a linear simplex the height function in the foliation chart yields a function in $\Delta$ with one maximum and one minimum in two vertices and no critical points elsewhere. See Figure 2.
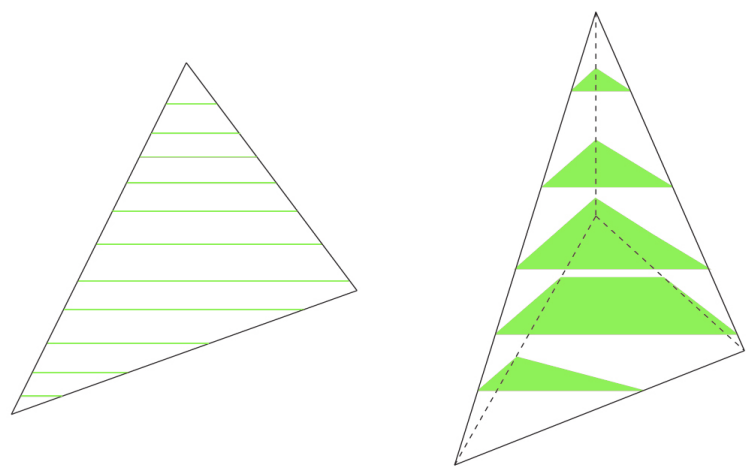

Fig. 2. A linear 2-simplex (left) and a linear 3-simplex (right) with their induced foliations (green).

A triangulation $\mathcal{T}$ of $V$ is adapted to the foliation $\mathcal{F}$ if all its simplices are linear with respect to $\mathcal{F}$. This corresponds to a distribution being in general position with respect to a triangulation. There always exists a triangulation on $V$ adapted to $\mathcal{F}$, see [Thurston, 1976]. The $i$-skeleton of $V$ with respect to this triangulation 
$\mathcal{T}$ is denoted by $V^{(i)}$.

In order to use Theorems 4.1 and 4.2 we require precise local models. The following lemma provides explicit models: these allow us to control the deformations that we obtain by applying the above $\mathrm{h}$-principles (with the suitable parameter spaces $L \subset K$ in each occasion).

Consider the standard closed 3-ball $D_{r}^{3} \subset \mathbb{R}^{3}$ of radius $r$ and fix a sequence of equators $\mathbb{S}_{r}^{0} \subset \mathbb{S}_{r}^{1} \subset \mathbb{S}_{r}^{2}$ respectively bounding closed flat disks $D_{r}^{1} \subset D_{r}^{2} \subset D_{r}^{3}$. For any interval $I$, the product foliation $\coprod_{t \in I}\{t\} \times D_{r}^{3}$ is denoted by $\mathcal{F}_{I \times D_{r}^{3}}$. Similarly, for a circle $\mathbb{S}^{1}$, we write $\mathcal{F}_{\mathbb{S}^{1} \times D_{r}^{3}}=\coprod_{t \in \mathbb{S}^{1}}\{t\} \times D_{r}^{3}$. When we omit the radius $r$, we will usually mean $r=1$, although in most cases any different $r>0$ might be achieved by a simple rescaling. Similarly, $I$ will usually denote the interval $[0,1]$. We can state the main lemma of this Subsection:

Lemma 4.3. Consider a triangulation $\mathcal{T}$ adapted to $(V, \mathcal{F})$, an index $j \in\{0, \ldots, 4\}$, a subset $G \subset V^{(j)}$ and a simplex $\sigma \in V^{(j)} \backslash G$. There exists an embedding $\phi_{\sigma}=\phi: I \times D^{3} \rightarrow V$ satisfying the following properties:

a. $\sigma \subset \operatorname{im}(\phi), \operatorname{im}(\phi)$ is contained in a small neighborhood of $\sigma$ and $\phi^{*} \mathcal{F}=\mathcal{F}_{I \times D^{3}}$.

b. There exist $\mathrm{Op}\left(V^{(j-1)} \cup G\right) \subset V, \mathrm{Op}(\partial I) \subset I$ and $\mathrm{Op}\left(\mathbb{S}^{j-2}\right) \subset D^{3}$ such that:

- For $j=0: \phi^{-1}(\operatorname{Op}(G))=\emptyset$,

- For $j=1: \phi^{-1}\left(\mathrm{Op}\left(V^{(0)} \cup G\right)\right)=\mathrm{Op}(\partial I) \times D^{3}$,

- For $j=2,3,4: \phi^{-1}\left(\mathrm{Op}\left(V^{(j-1)} \cup G\right)\right)=\left(\mathrm{Op}(\partial I) \times D^{3}\right) \cup\left(I \times \mathrm{Op}\left(\mathbb{S}^{j-2}\right)\right)$.

See Figure 3 for a schematic representation of the statement.

Proof. Consider an embedding $i: D^{j} \longrightarrow V$ of a closed $j$-disk extending $\sigma$ such that $i^{-1}(\partial \sigma)$ is arbitrarily close to $\partial D^{j}$. Since the triangulation $\mathcal{T}$ is adapted to $\mathcal{F}$, after a small isotopy, we can suppose that $i^{*} \mathcal{F}$ foliates the disk $D^{j}$ horizontally. In order to construct the embedding $\phi$ we use a normal frame along $i\left(D^{j}\right)$ which is contained in $\mathcal{F}$. Then the exponential map (and rescaling) yields an embedding

$$
\phi: D^{j} \times D^{4-j} \longrightarrow V,\left.\quad \phi\right|_{D^{j} \times\{0\}}=i
$$

such that $\sigma \subset \operatorname{im}(\phi)$ and $\operatorname{im}(\phi)$ is arbitrarily close to $\sigma$. This map can be understood as an embedding of $I \times D^{3}$ and it satisfies $\phi^{*} \mathcal{F}=\mathcal{F}_{I \times D^{3}}$. This is a foliation chart and for $j=0$ the statement follows.

Suppose that $j \neq 0$, let us detail the neighborhoods appearing in the statement. By construction $\phi\left(I \times D^{3}\right)$ is $C^{0}$-close to $\sigma$, hence any other simplex $\tau$ intersecting $\phi\left(I \times D^{3}\right)$ shares a face with $\sigma$. In particular, there 


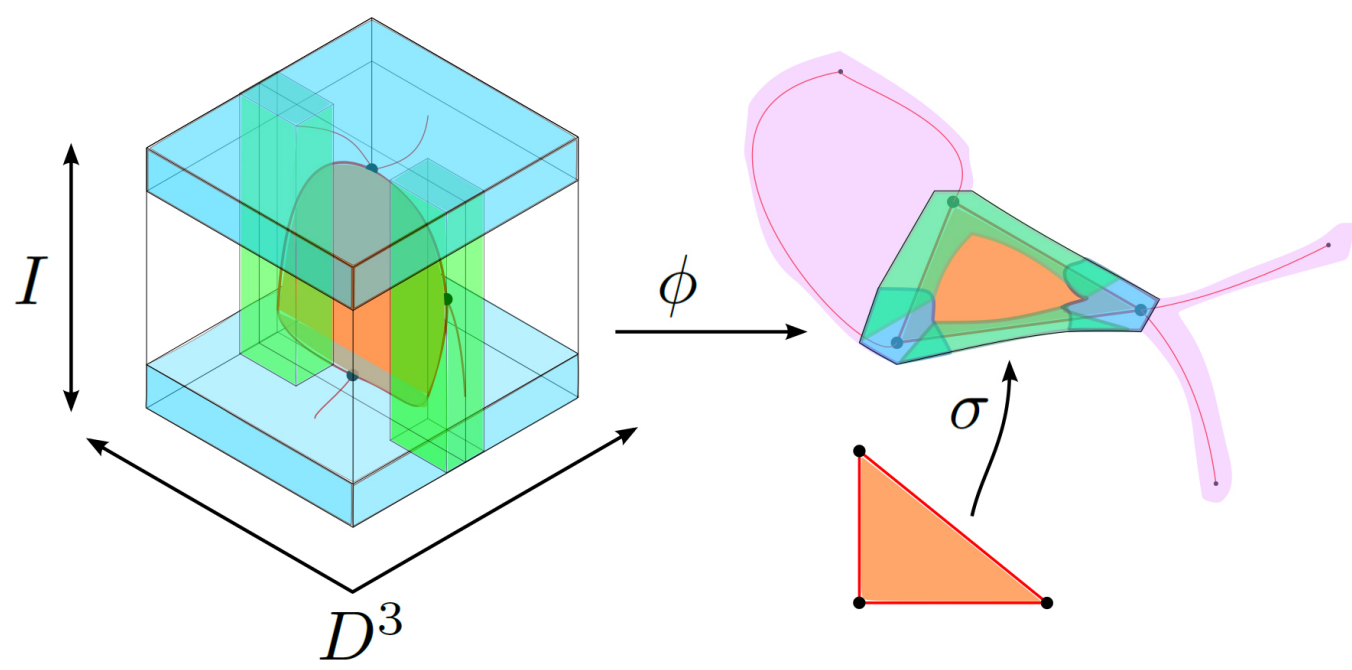

Fig. 3. Statement of Lemma 4.3 for the case $j=2$. The figure on the left depicts the local model and the one on the right a neighbourhood of its image in the manifold. The simplex $\sigma$ is depicted in orange, with edges in red. The neighbourhood $\operatorname{Op}\left(V^{(1)} \cup G\right)$ is colored in pink, and in this example it covers a whole simplex $\tau \in G$, to the left of $\sigma$, and two edges connected to the rightmost vertex of $\sigma$. The subsets $\operatorname{Op}(\partial I) \times D^{3}$ (blue) and $I \times \mathrm{Op}\left(\mathbb{S}^{0}\right)$ (green) cover the intersection of $\mathrm{Op}\left(V^{(1)} \cup G\right)$ with the image of the local model.

exists a neighborhood $\operatorname{Op}(\tau)$ such that $\mathrm{Op}(\tau) \cap \phi\left(I \times D^{3}\right) \subset \mathrm{Op}(\partial \sigma)$. Then $\operatorname{Op}\left(V^{(j-1)} \cup G\right)$ and $\mathrm{Op}(\partial \sigma)$ can be chosen so that $\mathrm{Op}\left(V^{(j-1)} \cup G\right) \cap \mathrm{Op}(\sigma)=\phi(\mathrm{Op}(\partial \sigma))$.

Note now that the preimage of the boundary $\phi^{-1}(\partial \sigma)$ is arbitrarily close to $\partial D^{j} \times\{0\}=\partial I \times D^{j-1} \cup I \times \mathbb{S}^{j-2}$ and thus we may suppose $\phi^{-1}(\mathrm{Op}(\partial \sigma))=\mathrm{Op}\left(\partial D^{j} \times\{0\}\right)$. By taking very small times for the exponential map above, it can be assumed that $\phi\left(\partial I \times D^{3}\right)$ is an arbitrarily small neighbourhood of the minimum and maximum. Then $\mathrm{Op}\left(\partial D^{j}\right)$ can be taken to be of the special form $\left(\partial I \times D^{3}\right) \cup\left(I \times \mathrm{Op}\left(\mathbb{S}^{j-2}\right)\right)$, proving the claim.

This a strictly topological result, not related to contact topology. Lemma 4.3 is used to apply the h-Principles in Sections 4.1 and 4.2 with a controlled choice of spaces $L \subset K$. In particular, it is needed in Proposition 4.6, which constructs a foliated contact structure on the 3-skeleton, and in Theorem 1.1, where we finally deal with the 4-skeleton.

Theorem 1.1 holds for any pair $\left(V^{4}, \mathcal{F}^{3}\right)$. It is however shorter (and illustrative) to consider the case of a taut foliation $\mathcal{F}$. This hypothesis simplifies the argument and Theorem 1.1 is first proved in Section 5 for this case. Let us state two technical brief lemmas that we use in the proof.

Lemma 4.4. Let $\mathcal{F}$ be a taut foliation on $V$. Consider a 4 -simplex $\sigma \in V^{(4)}$ and a map $\phi: I \times D^{3} \longrightarrow V$ provided by Lemma 4.3 . There exists a map $\gamma_{\sigma}:[0,2] /\{0 \sim 2\}=\mathbb{S}^{1} \longrightarrow V$ transverse to $\mathcal{F}$ such that: 
- $\gamma_{\sigma}(t)=\phi(t, 0)$ for $t \in I$,

- $\gamma_{\sigma}(t) \in \operatorname{Op}\left(V^{(3)}\right)$ for $t \in \mathbb{S}^{1} \backslash I$.

Proof. Since the foliation $\mathcal{F}$ is taut and $V$ connected, there exists a positively transverse path

$$
l:[1,2] \longrightarrow V, \quad l(1)=\phi(1,0), \quad l(2)=\phi(0,0) .
$$

The path $l$ can be assumed to lie in (a neighborhood of) the 3-skeleton $V^{(3)}$ up to isotopy. We can concatenate $\phi(t, 0)$ for $t \in(0,1)$ and $l(t)$ for $t \in[1,2]$, and smooth the resulting map in order to obtain the required map $\gamma_{\sigma}$.

A generic choice of maps $\phi_{\sigma}$ implies that:

Corollary 4.5. Given a collection $G \subset V^{(4)}$ there exists a collection $\left\{\gamma_{\sigma}\right\}_{\sigma \in G}$ of pairwise disjoint paths satisfying the properties of Lemma 4.4 .

Both Lemma 4.4 and Corollary 4.5 are used in Section 5 to conclude Theorem 1.1 in the case of a taut foliation. Their analogues for the general case of Theorem 1.1 are stated in Section 6. The techniques used also apply to prove that Theorem 1.1 holds for open manifolds. The details of this are left to the reader. Note first that all the statements from this section hold in the open case.

\subsection{3-skeleton}

The argument for Theorem 1.1 is constructive on the simplices of a triangulation of $V$. It begins with the lower dimensional skeleta and concludes with the construction of a foliated contact structure on the simplices of $V^{(4)}$. This is a commonly used strategy for flexibility results, for instance [Eliashberg, 1989]. It is often the case that the deformation required in the skeleta below the top-dimensional strata can be easily achieved.

Proposition 4.6. Let $(\xi, J)$ be a foliated almost contact structure on $(V, \mathcal{F})$. There exists a homotopy $\left\{\left(\xi_{t}, J_{t}\right)\right\}$ of foliated almost contact structures such that $\left(\xi_{0}, J_{0}\right)=(\xi, J)$ and $\left(\xi_{1}, J_{1}\right)$ is a contact foliation on $V^{(3)}$.

Proof. Consider the 0 -skeleton $V^{(0)}$. Lemma 4.3 gives disjoint foliation charts $\phi: I \times D^{3} \longrightarrow \mathrm{Op}(\sigma)$ near each 0 -simplex $\sigma$. The foliated almost contact structure $\left(\phi^{*} \xi_{0}, \phi^{*} J_{0}\right)$ can be considered as a 1-parametric family $\left(\xi_{t}, J_{t}\right)$ of almost contact structures in the disc $D^{3}$. Then we can apply Theorem 4.1 with $K=I, V=D^{3}$, $U=D_{0.5}^{3}, L=\emptyset$ and $A=\emptyset$ and obtain a foliated contact structure in a neighbourhood of the 0 -skeleton. 
We fix an index $j \in\{1,2,3\}$ and apply induction on the set of simplices of the $j$-skeleton $V^{(j)}$. Given a simplex $\sigma$, Lemma 4.3 provides an embedding $\phi: I \times D^{3} \longrightarrow \mathrm{Op}(\sigma)$ and we can consider a small $\delta>0$ and an embedding $\phi: I \times D_{1-\delta}^{3} \longrightarrow V$ which also conforms to the properties of Lemma 4.3. Then Theorem 4.1 applies with $K=I$, $V=D^{3}, U=D_{1-\delta}^{3}, L=\mathrm{Op}(\partial I), A=\mathbb{S}_{1-\delta}^{j-2}$. This inductive procedure constructing the contact foliated structure (relative to the previous step) applied to the 1,2 and 3-skeleton implies the statement.

In the next two sections we prove the theorem. First, it is concluded for the case of a taut foliation $\mathcal{F}$ in Section 5. Then Section 6 shortly adapts Section 4.3 and proves the general result stated in Section 2.

\section{Taut Case}

In this section we prove Theorem 1.1 in the case where the foliation $\mathcal{F}$ is taut. We fix a triangulation $\mathcal{T}$ of $V$ adapted to the taut foliation $\mathcal{F}$. Given a foliated almost contact structure $(\xi, J)$, its associated pair is denoted by $(\beta, \alpha)$.

The proof consists of two steps. The first step, Proposition 5.1, provides an appropriate normal form for the foliated almost contact structure (or rather its defining form) in a neighborhood of a 4-simplex. The second step, Proposition 5.2, uses this local model in order to insert a foliated family of overtwisted disks.

In order to obtain a normal form for the defining form of the foliated almost contact structure we choose a convenient trivialization. This method has been used in the contact setup, see [Casals, Presas, 2013]. The following Proposition adapts the technique to the foliated framework.

Proposition 5.1. Suppose $(\xi, J)$ is a foliated contact structure on $\operatorname{Op}\left(V^{(3)}\right)$ and consider the set of maps $\left\{\gamma: \mathbb{S}^{1} \longrightarrow V\right\}$ given by Corollary 4.5 applied to $G=V^{(4)}$. For each such $\gamma$, there exists an embedding

$$
\phi_{\gamma}: \mathbb{S}^{1}(t) \times D_{R}^{3}(z, r, \theta) \longrightarrow V \quad \text { for some radius } R>0 \text { such that } \phi(\cdot ; 0)=\gamma(\cdot)
$$

and satisfying the following properties:

a. $\phi^{*} \mathcal{F}=\mathcal{F}_{\mathbb{S}^{1} \times D}$

b. $\phi^{-1}\left(\mathrm{Op}\left(V^{(3)}\right)=I_{2} \times D_{R}^{3}\right.$, for some open interval $I_{2} \subset \mathbb{S}^{1}$,

c. $\phi^{*} \xi=\operatorname{ker}(\widetilde{\alpha}) \cap \phi^{*} \mathcal{F}$, where $\widetilde{\alpha}=d z+f(t ; z, r, \theta) d \theta$ and $f(t ; 0)=0$.

Also, there exists a small $\delta>0$ such that the bounds

$$
\partial_{r} f(t ; z, r, \theta)>2 \delta r, \quad f(t ; z, r, \theta)>\delta r^{2},
$$


hold for all $t$ such that $\gamma(t) \in \mathrm{Op}\left(V^{(3)}\right)$.

Proof. Consider a path $\gamma_{\sigma}$ for a fixed $\gamma \in G$ and a set $\{T, X, Y, Z\}$ of commuting vector fields trivializing $\mathrm{Op}\left(\gamma_{\sigma}\right)$ such that:

- $T$ preserves the foliation $\left.\mathcal{F}\right|_{\mathrm{Op}\left(\gamma_{\sigma}\right)}$ and its flowlines are circles of period 1. In particular, $\gamma_{\sigma}$ is one such flowline,

- the triple $\{X, Y, Z\}$ trivializes $\left.T \mathcal{F}\right|_{\mathrm{Op}\left(\gamma_{\sigma}\right)}$,

- $\alpha(Z)>0$ and $\alpha(t ; 0)(X)=\alpha(t ; 0)(Y)=0$.

This trivialization integrates to an embedding $\psi: \mathbb{S}^{1}(t) \times D_{\rho}^{3}(x, y, z) \longrightarrow V$ for some small $\rho>0$. The pull-back of the defining form in these coordinates reads $\psi^{*} \alpha=h_{0} d z+h_{1} d x+h_{2} d y$, where $h_{0}, h_{1}, h_{2} \in C^{\infty}\left(\mathbb{S}^{1} \times D_{\rho}^{3}\right)$ satisfy $h_{0}>0$ and $h_{1}(t, 0)=h_{2}(t, 0)=0$. Since $h_{0}$ is positive, we can rescale the form by $1 / h_{0}$ and then changing to polar coordinates yields a local expression of the form $\psi^{*} \alpha=d z+f d r+g d \theta$, where the equality is up to a conformal factor, $f=O(r)$ and $g=O\left(r^{2}\right)$.

In order to conclude the statement we need to erase the $d r$ factor, or equivalently, to find a radial coordinate belonging to the distribution. Consider the fibration

$$
\begin{aligned}
\{t\} \times D_{\rho}^{3} & \longrightarrow D_{\rho}^{2} \\
(t ; z, r, \theta) & \longmapsto(r, \theta),
\end{aligned}
$$

endowed with the Ehresmann connection $\operatorname{ker}(\alpha) \bigcap \operatorname{ker} d t$. The radial vector field $\partial_{r}$ on the base $D_{\rho}^{2}$ lifts to $h_{r}=\partial_{r}-f \partial_{z}$. For fixed $t_{0}, \theta_{0}$ and $z_{0}$ the equation

$$
z^{\prime}(r)=-f\left(t_{0} ; z(r), r, \theta_{0}\right), \quad z(0)=z_{0}
$$

is an ODE whose solution $F_{\left(t_{0} ; z_{0}, \theta_{0}\right)}(r)$ is defined for small time $\rho^{\prime}>0 . \rho^{\prime}$ can be assumed to be independent of the choice of parameters, since the ODE depends smoothly on them. After possibly reparametrising in the $z$ coordinate, the new coordinate system is given precisely by:

$$
\begin{aligned}
\Phi: \mathbb{S}^{1} \times D_{\rho^{\prime}}^{3} & \longrightarrow \mathbb{S}^{1} \times D_{\rho}^{3} \\
(t ; z, r, \theta) & \longmapsto\left(t ; F_{(t ; z, \theta)}(r), r, \theta\right) .
\end{aligned}
$$


The defining form $\alpha$ is expressed in these coordinates as $(\psi \circ \Phi)^{*} \alpha=d z+f d \theta$ for some (other) smooth function $f$. By shrinking $\operatorname{Op}\left(V^{(3)}\right)$, the preimage $\Phi^{-1}\left(\mathrm{Op}\left(V^{(3)}\right)\right)$ can be supposed to be of the form $I_{2} \times D_{\rho^{\prime}}^{3}$. Finally, the bounds on $f$ readily follow from $\alpha$ being a foliated contact form in $\operatorname{Op}\left(V^{(3)}\right)$. Fix $\rho^{\prime}>R>0$.

Remark 2. Given $\sigma \in V^{(4)}$, the maps $\left.\phi_{\sigma}\right|_{I \times D_{R}^{3}}$ and $\left.\phi_{\gamma_{\sigma}}\right|_{I \times D_{R}^{3}}$ do not necessarily agree. However, since the interval $I$ is contractible and $R>0$ can be assumed to be sufficiently small so that $\operatorname{im}\left(\phi_{\sigma}^{-1} \circ \phi_{\gamma_{\sigma}}\right)$ is disjoint from $\mathrm{Op}\left(I \times \mathbb{S}^{j-2}\right), \phi_{\sigma}$ can be deformed near $I \times\{0\}$ so that both embeddings agree in $I \times D_{R}^{3}$.

The previous Proposition provides a local description of the foliated almost contact structure. We will use such model to deform the (almost) contact structure on the leaves to overtwisted almost contact structures. This is the content of the following Proposition.

Proposition 5.2. Suppose $(\xi, J)$ is a foliated contact structure on $\operatorname{Op}\left(V^{(3)}\right)$. There exists a homotopy of foliated almost contact structures $\left(\xi_{s}, J_{s}\right)$ such that:

a. $\left(\xi_{0}, J_{0}\right)=(\xi, J)$ and $\left(\xi_{1}, J_{1}\right)$ is a foliated contact structure on $\mathrm{Op}\left(V^{(3)}\right)$.

b. Given $\sigma \in V^{(4)}, \phi_{\gamma}^{*} \xi_{1}$ is foliated contact in $\operatorname{Op}(I \times\{0\})$.

c. If we consider $\phi_{\gamma}^{*} \xi_{1}$ as a family $\left\{\xi_{t}\right\}_{t \in I}$ of contact structures on $D^{3}$, there exists a disc $\Delta \subset D^{3}$ which is overtwisted $\forall \xi_{t}$.

Proof. There are two steps. First, find a foliated Darboux normal form in a neighborhood of a 1-parametric family of knots. Second, perform a Lutz twist along each of them.

Let us first deform the foliated (almost) contact structure provided by Proposition 5.1 in order for it to be standard near the core. This procedure is done for each 4-simplex $\sigma$ and their corresponding map $\gamma_{\sigma}$ as obtained in Corollary 4.5. In the local model $\phi_{\gamma}: \mathbb{S}^{1} \times D_{R}^{3} \longrightarrow V$ of Proposition 5.1 the foliated defining form reads $\phi_{\gamma}^{*} \alpha=d z+f d \theta$ on each leaf. Consider a decreasing smooth cut-off function $\chi:[0, R] \longrightarrow[0,1]$ such that:

$$
\chi(r)=1 \text { in }[0, R / 3] \text { and } \chi(r)=0 \text { in }[2 R / 3, R] .
$$

This cut-off is used in order to smoothly modify $f$ to the local model $\delta r^{2}$. Indeed, consider the function $\widetilde{f} \in C^{\infty}\left(\mathbb{S}^{1} \times D_{R}^{3}\right)$ defined by $\widetilde{f}=\delta r^{2} \cdot \chi(r)+f \cdot(1-\chi(r))$. The bounds in Proposition 5.1 imply that

$$
\partial_{r} \tilde{f}=\left(\chi^{\prime}\left(\delta r^{2}-f\right)\right)+\left(2 r \delta \chi+\frac{\partial f}{\partial r}(1-\chi)\right)>0
$$


in $\phi^{-1}\left(\mathrm{Op}\left(V^{(3)}\right)\right)$.

Since the 1 -form $\left(\phi_{\gamma}\right)_{*}(d z+\widetilde{f} d \theta)$ agrees with $\alpha$ near the boundary of $\phi_{\gamma}\left(\mathbb{S}^{1} \times D_{R}^{3}\right)$, it extends to a global 1 -form $\widetilde{\alpha}$. Then, the leafwise defined plane field $\widetilde{\xi}=\operatorname{ker}(\widetilde{\alpha})$ is a foliated almost contact distribution which is, by construction, homotopic to $\xi$. This concludes the first step.

For a radius $\rho>0$ sufficiently small, consider the embedded torus

$$
\begin{aligned}
\eta: \mathbb{S}^{1} \times \mathbb{S}^{1} & \longrightarrow V \\
(t, \theta) & \longmapsto \eta(t, \theta)=\phi_{\gamma}(t, 0, \rho, \theta) .
\end{aligned}
$$

It should be considered as an $\mathbb{S}^{1}$-family of loops transverse to the (almost) contact structures on the leaves. We can now deform $\widetilde{\xi}$ by performing a full Lutz twist along each curve $\eta_{t}(\theta)=\eta(t, \theta)$. This procedure boils down to replacing the standard contact model along the transverse knot $\eta_{t}(\theta)$ by a model in which the contact structure makes a full turn, see [Geiges, 2008, Section 4.3]. It is also proven in said reference that the deformation is homotopic to the original structure as almost contact distributions. This yields an almost contact distribution $\left(\xi_{1}, J_{1}\right)$ such that for each $\theta \in \mathbb{S}^{1}$, the almost contact structure $\xi_{1}$ has a 1 -parametric family of overtwisted $\operatorname{discs}\left\{\Delta_{t}\right\}_{t \in \mathbb{S}^{1}}$ centered at the points $\phi(t, 0, \rho, \theta)$. The resulting foliated almost contact structure satisfies the properties of the statement.

The results from Section 4 and the previous Proposition are enough to conclude Theorem 1.1 in the case that $\mathcal{F}$ is a taut foliation.

Proof of Theorem 1.1 (Taut Case). We first apply Proposition 4.6 to the foliated almost contact structure $(\xi, J)$ in order to obtain a foliated almost contact structure which is foliated contact on $\mathrm{Op}\left(V^{(3)}\right)$. This foliated almost contact structure satisfies the hypotheses of Proposition 5.2 and thus there exists a deformation to a foliated almost contact structure which is still contact near $\mathrm{Op}\left(V^{(3)}\right)$ and each leafwise almost contact structure on the 4 -simplices has an overtwisted disk. Hence, we can apply Proposition 4.2 to each 4 -simplex with $K=I$, $V=D^{3}, A=\mathbb{S}^{2}, L=\partial I$ and the overtwisted disk $\Delta$ (where the neighborhoods are obtained using Proposition $5.2)$. This yields a deformation of foliated almost contact structures to a foliated contact structure $\left(\xi_{1}, J_{1}\right)$.

This concludes Theorem 1.1 for a taut foliation. The proof does not directly apply to the case of a general foliation $\mathcal{F}$, however the strategy can be modified. This is explained in the subsequent section. 


\section{Proof of the General Case}

\subsection{Proof of Theorem 1.1}

\subsubsection{Preliminaries}

In the case that $\mathcal{F}$ is a taut foliation, the argument in Section 5 applies. In order to adapt the proof for a general foliation we introduce the notion of a vanishing Lutz twist. We fix a triangulation $\mathcal{T}$ of $V$ adapted to the foliation $\mathcal{F}$ and denote by $(\beta, \alpha)$ a pair associated to the foliated almost contact structure $(\xi, J)$.

Note that Proposition 4.6 applies to any almost contact foliation. Hence, we suppose that $\xi$ is a foliated contact structure in $\mathrm{Op}\left(V^{(3)}\right)$. We first state two lemmas as in Section 4.3 and then introduce the vanishing family of Lutz twists in Subsection 6.1.2. This allows us to conclude Theorem 1.1 for any foliated almost contact structure on any codimension-1 foliated $4-$ fold $(V, \mathcal{F})$.

The arguments for the following two results are closely related to the proofs of Lemma 4.4 and Proposition 5.1. We include the corresponding statements and leave the details of the proofs to the reader. $\widetilde{\mathrm{Op}}(X)$ denotes an arbitrarily small neighbourhood of the set $X$ that is chosen to be relatively compact within some $\operatorname{Op}(X)$.

Lemma 6.1. Consider a 4-simplex $\sigma \in V^{(4)}$ and the map $\phi_{\sigma}$ (as in Lemma 4.3). There exist a sequence of open intervals $I \subsetneq I_{1} \subsetneq I_{2} \subsetneq I_{3}$ and a map $\gamma_{\sigma}: I_{3} \longrightarrow V$ transverse to $\mathcal{F}$ such that:

$$
\begin{aligned}
& \text { - } \gamma_{\sigma}(t)=\phi(t ; 0) \text { for } t \in I, \\
& \text { - } \gamma_{\sigma}\left(I_{1} \backslash I\right) \subset \widetilde{\mathrm{Op}}\left(V^{(3)}\right), \gamma_{\sigma}\left(I_{2} \backslash I_{1}\right) \subset \mathrm{Op}\left(V^{(3)}\right) \backslash \widetilde{\mathrm{Op}}\left(V^{(3)}\right) \text { and } \gamma_{\sigma}\left(I_{3} \backslash I_{2}\right) \subset V \backslash \mathrm{Op}\left(V^{(3)}\right) \text {. }
\end{aligned}
$$

Also, $\gamma_{\sigma}\left(I_{3}\right)$ and $\gamma_{\tau}\left(I_{3}\right)$ are disjoint if $\tau \in V^{(4)}$ is different from $\sigma$.

Lemma 6.2. Consider a map $\gamma_{\sigma}: I_{3} \longrightarrow V$ (provided by Lemma 6.1). There exists an embedding

$$
\kappa_{\gamma_{\sigma}}: I_{3} \times D_{R}^{3} \rightarrow V
$$

for some small radius $R>0$ such that $\kappa_{\gamma_{\sigma}}(\cdot ; 0)=\gamma_{\sigma}(\cdot)$ and conforming the following properties:

a. $\kappa_{\gamma_{\sigma}}^{*} \mathcal{F}=\mathcal{F}_{I_{3} \times D}$,

b. the map $\left.\phi_{\sigma}\right|_{I \times D_{R}^{3}}$ can be perturbed so that it agrees with $\left.\kappa_{\gamma_{\sigma}}\right|_{I \times D_{R}^{3}}$.

Also, there exists an almost contact structure $(\widetilde{\xi}, \widetilde{J})$ homotopic to $(\xi, J)$ which is foliated contact on $\operatorname{Op}\left(V^{(3)}\right)$ such that:

$$
\kappa_{\gamma_{\sigma}}^{*} \widetilde{\xi}=\operatorname{ker}(\widetilde{\alpha}) \cap \kappa_{\gamma_{\sigma}}^{*} \mathcal{F}, \quad \text { where } \widetilde{\alpha}=d z+r^{2} d \theta, \quad \forall t \in I_{2}, \forall \sigma \in V^{(4)}
$$


$\left(I_{3} \backslash I_{2}\right) \times D_{R}^{3}$ is the region in which the interpolation between the old almost contact structure $\xi$ and the new one $\widetilde{\xi}$ takes place.

\subsubsection{Vanishing Lutz twist}

In the case of a general foliation $\mathcal{F}$ we cannot insert a transverse (circle) family of overtwisted disks intersecting every leaf. We solve this by considering a transverse interval assigned to each 4 -simplex and performing a compactly supported or vanishing Lutz twist. Let us define this.

Consider the almost contact structure $\widetilde{\xi}$ obtained in Lemma 6.2. Lemma 6.2 states that the curve $\gamma_{\sigma}$ associated to the 4 -simplex $\sigma$ has a local model $\kappa_{\gamma_{\sigma}}$ in which the pullback structure $\kappa_{\gamma_{\sigma}}^{*} \widetilde{\xi}$ is $t$-invariant and contact on $I_{2} \times D_{R}^{3}$. This $t$-invariant contact structure in the $\operatorname{disc} D_{R}^{3}$ is given by $\operatorname{ker}\left(d z+r^{2} d \theta\right)$. Denote it by $\xi^{0}$. As was described in the previous section, one fixes now a loop $K \subset D_{R}^{3}$ transverse to $\xi^{0}$ and performs a Lutz twist along it. This yields a new contact structure $\xi^{1}$ in $D_{R}^{3}$ that agrees with $\xi^{0}$ away from $K$. As almost contact structures they are actually homotopic - but not homotopic through contact structures - and one can denote the homotopy by $\xi^{s} . \xi^{s}$ can be assumed to agree with $\xi^{0}$ close to $\partial D_{R}^{3}$.

Consider a cut-off smooth function $\chi: I_{2} \longrightarrow[0,1]$ such that:

- $\chi(t)=1$ for $t \in I_{1}$ and $\chi(t)=0$ for $t$ near $\partial I_{2}$,

- $\chi(t)$ is monotone in the two components of $I_{2} \backslash I_{1}$.

Definition 6.3 (Vanishing Family of Lutz twists). The distribution $\xi^{\chi(t)} \cap \operatorname{ker}(d t)$ defined in $I_{2}(t) \times D_{R}^{3}$ is called a vanishing family of Lutz twists along the segment $I_{2} \times\{0\}$.

Note that $\xi^{\chi(t)} \cap \operatorname{ker}(d t)$ is a foliated almost contact structure in $I_{2} \times D_{R}^{3}$ that agrees with the $t$-invariant structure $\xi^{0} \cap \operatorname{ker}(d t)$ close to the boundary $\left(\partial I_{2} \times D_{R}^{3}\right) \bigcup\left(I_{2} \times \partial D_{R}^{3}\right)$. Given a foliated almost contact structure $\widetilde{\xi}$ with local model $\kappa_{\gamma_{\sigma}}^{*} \widetilde{\xi}=\xi^{0} \cap \operatorname{ker}(d t)$ we will say that replacing $\left(\kappa_{\gamma_{\sigma}}\right)_{*}\left(\xi^{0} \cap \operatorname{ker}(d t)\right)$ by $\left(\kappa_{\gamma_{\sigma}}\right)_{*}\left(\xi^{\chi(t)} \cap \operatorname{ker}(d t)\right)$ is applying a vanishing Lutz twist to $\widetilde{\xi}$ along the segment $\gamma_{\sigma}$.

Proof of Theorem 1.1. By the discussion above we can assume that we are already in the conclusions of Lemma 6.2. Write $\widetilde{\xi}$ for the almost contact structure it yielded. For every 4 -simplex $\sigma$ we consider the associated curve $\gamma_{\sigma}: I_{3} \rightarrow V$ and we perform a vanishing Lutz twist along it. Denote this new structure by $\widetilde{\xi}^{\prime}$.

Since $\left.\chi\right|_{I}=1$, this means that now we have a family of overtwisted disks along the curve $\gamma_{\sigma} \cap \operatorname{im}\left(\phi_{\sigma}\right)$-in the local model, $\xi^{\chi(1)}=\xi^{1}$ was the contact structure that had a Lutz twist. Further, the structure $\widetilde{\xi}^{\prime}$ is still contact in $\widetilde{\mathrm{Op}}\left(V^{(3)}\right)$, since $\left.\chi\right|_{I_{1}}=1$ as well. Now Proposition 4.2 can be applied to $\widetilde{\xi}^{\prime}$ in each 4 -simplex to yield the result, just like in the taut case. 


\subsection{Proof of Corollary $\mathbf{1 . 2}$}

We describe the proof of Theorem 1.1 for continuous parametric families of almost contact structures $\left\{\left(\xi_{t}, J_{t}\right)\right\}_{t \in P}$, for an arbitrary compact set of parameters $P$. The steps carried out in the previous subsection admit a parametric version, let us briefly discuss it.

1. Since the parameter space $P$ is compact, the neighbourhoods $\operatorname{Op}\left(V^{(j-1)} \cup G\right)$ (introduced in Lemma 4.3) and $\mathrm{Op}\left(V^{(j)}\right)$, in which the structures are already foliated contact, can be assumed not to depend on $t \in P$. Therefore, the results contained in Subsection 4.3 refer only to the fixed foliation $\mathcal{F}$ and thus the addition of parameters is trivial. The same reasoning applies to Lemma 6.1.

2. In the zero skeleton we proceed as in Proposition 4.6, by setting $V=D^{3}, U=D_{0.5}^{3}, K=I \times P, L=\emptyset$, and $A=\emptyset$, and applying Theorem 4.1. For the case $j \in\{1,2,3\}$, define $V=D^{3}, U=D_{1-\delta}^{3}, K=I \times P$, $L=\mathrm{Op}(\partial I) \times P$ and $A=\mathbb{S}_{1-\delta}^{j-2}$.

3. The deformation of $\xi$ to $\widetilde{\xi}$ provided by Lemma 6.2 , to which one applies the vanishing Lutz twist, can be reproduced parametrically. Indeed, the curves $\gamma$ around which the deformation is done do not depend on $t \in P$ and following the proof shows that a $P$-parametric family of embeddings $\kappa_{\gamma, t}: I_{3} \times D_{R}^{3} \rightarrow V$ (as in Lemma 6.2) can be constructed so that $R$ does not depend on $t$ and the pullback of the deformation $\widetilde{\xi}_{t}$ of $\xi_{t}$ is given by the kernel of $d z+r^{2} d \theta$.

4. Then, the vanishing Lutz twist can be produced parametrically along a fixed family of intervals, depending on $t \in P$. In each local model $I \times D^{3}$ this yields an overtwisted disc $\Delta_{t} \subset D^{3}$. Then, an extension of Theorem 4.2 for the case of a parametric family of overtwisted disks yields the result considering $K=I \times P, V=D^{3}, U=\mathrm{Op}\left(\mathbb{S}^{2}\right), L=\partial I \times P$ and the overtwisted disks $\Delta_{t}$.

Let us mention some possible extensions of Theorem 1.1. First, the hypotheses on orientability and coorientability can be weakened. The same argument as in the orientable and coorientable case holds for the non-orientable and non-coorientable cases by taking double covers appropriately. Second, Theorem 1.1 also holds for open 4-folds. Finally, we believe that the argument can be modified to apply to the case of foliations of higher codimension (the essential step being an appropriate construction of the vanishing family of Lutz twists).

\section{Acknowledgements}

The authors are thankful to V. Ginzburg, R. Loja Fernandes and E. Miranda for their remarks and suggestions during the development of this paper. The authors would also like to thank K. Niederkrüger, D. Pancholi, D. Peralta-Salas and A. Rechtman for useful discussions. The authors are supported by the Spanish National Research Project MTM2010-17389. The present work is part of the authors activities within CAST, a Research 
Network Program of the European Science Foundation. The article was partially written during the stay of the first author at Stanford University, he is grateful to Y. Eliashberg for his hospitality.

\section{References}

A. Abouqateb, M. Boucetta, "The modular class of a regular Poisson manifold and the Reeb class of its symplectic foliation." (English, French summary) C. R. Math. Acad. Sci. Paris 337 (2003), no. 1, 61-66.

F. Alcalde-Cuesta, "Préquantification de certaines variétés de Poisson". Geometric study of foliations. (Tokyo, 1993), 123-143. World Sci. Publ., River Edge, NJ, 1994.

F. Alcalde-Cuesta and G. Hector, "Intégration symplectique des variétés de Poisson régulières." Israel J. Math., 90 (1995), no. 1-3, 125-165.

M. Bertelson, "A h-principle for open relations invariant under foliated isotopies." J. Symplectic Geom. 1, no. 2 (2002): 369-425.

M.S. Borman, Y. Eliashberg and E. Murphy, "Existence and classification of overtwisted contact structures in all dimensions." arXiv:1404.6157.

M. Crainic and R. Fernandes, "Integrability of Poisson brackets." J. Differential Geom. 66 no. 1 (2004): 71-137.

M. Crainic and R. Fernandes, "Stability of symplectic leaves." Invent. Math. 180 no. 3 (2010): 481-533.

R. Casals and F. Presas, "A remark on the Reeb flow for spheres." To appear in J. Symp. Geom.

Y. Eliashberg, "Classification of overtwisted contact structures on 3-manifolds." Invent. Math. 98 (1989): 623637.

Y. Eliashberg, S.S. Kim and L. Polterovich, "Geometry of contact transformations and domains: orderability versus squeezing." Geom. Topol. 10 (2006): 1635-1747.

Y. Eliashberg, and N. Mishachev. Introduction to the h-Principle. Graduate studies in Mathematics, Vol. 48, AMS publications (2002).

R. Fernandes, "Connections in Poisson geometry. I. Holonomy and invariants." J. Differential Geom. 54 (2000), n. 2, 303-365.

P. Frejlich, and R. Loja Fernandes. "An h-principle for symplectic foliations." Int. Math. Res. Not. no. 7 (2012): $1505-1518$.

P. Frejlich, D. Martínez Torres, and E. Miranda. "Symplectic topology of b-symplectic manifolds." arxiv:1312.7329 
K. Fukaya and K. Ono. "Arnold conjecture and Gromov-Witten invariant." Topology. 38 no. 5 (1999): 933-1048.

H. Geiges, An Introduction to Contact Topology, Cambr. Studies in Adv. Math. 109. Cambr. Univ. Press, 2008.

V. Guillemin, E. Miranda, and A.R. Pires. "Codimension one symplectic foliations and regular Poisson structures." Bull. Braz. Math. Soc. (N.S.) 42 no. 4 (2011):607-623.

M. Gromov, "Pseudo holomorphic curves in symplectic manifolds." Invent. Math. 82 (1985): 307-347.

M. Gromov, Partial Differential Relations, Ergeb. Math. Grenzgeb. 9 Springer-Verlag, 1986.

G. Hector, "Une nouvelle obstruction à l'intégrabilité des variétés de Poisson régulières." Hokkaido Math. J. 21 No. 1 (1992): 159-185.

G. Hector, E. Macías, and M. Saralegi. "Lemme de Moser feuilleté et classification des variétés de Poisson régulières." Publ. Mat. 33 no. 3 (1989): 423-430.

A. Ibort and D. Martínez Torres. "A new construction of Poisson manifolds." J. Symp. Geom. 2 No.1 (2003): 83-107.

A. Ibort and D. Martínez-Torres. "Approximately holomorphic geometry and estimated transversality on 2calibrated manifolds." C. R. Math. Acad. Sci. Paris 338 no. 9 (2004): 709-712.

E. Lerman, "Contact fiber bundles." J. Geom. Phys. 49 no. 1 (2004): 52-66.

P. Libermann, "Sous-variétés et feuilletages symplectiquement réguliers." Res. Notes in Math. 80 (1983):81-106.

G. Liu, and G. Tian. "Floer homology and Arnold conjecture." J. Diff. Geom. 49 (1998): 1-74.

R. Lutz, "Sur l'existence de certaines formes différentielles remarquables sur la sphère $\mathbb{S}^{3}$." C.R. Acad. Sci. Paris 270 (1970): 1597-1599.

I. Marcut, "Normal forms in Poisson geometry", arXiv.math:1301.4571.

J. Martinet, "Formes de contact sur les variétés de dimension 3." Proc. Liverpool Sing. Symp. II, Lect. Notes in Math. 209 (1971): 142-163.

D. Martínez Torres "Codimension-one foliations calibrated by nondegenerate closed 2-forms." Pacific J. Math. 261 no. 1 (2013): 165-217.

D. Martínez Torres "A higher dimensional generalization of taut foliations." arxiv:math/0602576

D. McDuff, and D. Salamon. Introduction to symplectic topology, Second edition. Oxford Mathematical Monographs. The Clarendon Press, Oxford University Press, New York, 1998. 
K. Mikami, "Godbillon-Vey classes of symplectic foliations." Pacific J. Math. 194 no. 1 (2000): 165-174.

A. del Pino, and F. Presas. "Foliated Weinstein conjecture." In preparation.

L. Polterovich, The geometry of the group of symplectic diffeomorphisms, Lectures in Mathematics ETH Zürich, Birkhäuser, 2001.

T. Rybicki, "On the flux homomorphism for regular Poisson manifolds." Proceedings of the 17th Winter School "Geometry and Physics" (Srn, 1997). Rend. Circ. Mat. Palermo (2) Suppl. No. 54 (1998), 9199.

D. Sullivan, "Cycles for the dynamical study of foliated manifold and complex manifolds." Invent. Math. 36 (1976): 225-255.

C. H. Taubes, "The Seiberg-Witten equations and the Weinstein conjecture." Geom. Topol. 11 (2007): 21172202.

W. P. Thurston, "Existence of codimension-one foliations." Ann. of Math. 104 (1976): 249-268.

I. Vaisman, Lectures on the geometry of Poisson manifolds, Progress in Mathematics, 118. Birkhäuser Verlag, 1994.

A. Weinstein, "Periodic orbits for convex Hamiltonian systems." Ann. of Math. 108 (1978): 507-518.

P. Xu, "Poisson cohomology of regular Poisson manifolds." Ann. Inst. Fourier (Grenoble) 42 (1992), n. 4, 967-988. 